\section{$\underset{\substack{\text { hommes } \\ \text { \& migrations }}}{ }$}

\section{Hommes \& migrations}

Revue française de référence sur les dynamiques

migratoires

$1309 \mid 2015$

Le 3 e âge des migrants

\title{
Aux origines de la revue Hommes \& Migrations
}

L'association Amana et la création des Cahiers nord-africains (1945-1965)

\section{Angéline Escafré-Dublet}

\section{(e) OpenEdition \\ 1 Journals}

\section{Édition électronique}

URL : http://journals.openedition.org/hommesmigrations/3101

DOI : 10.4000/hommesmigrations.3101

ISSN : 2262-3353

Éditeur

Musée national de l'histoire de l'immigration

\section{Édition imprimée}

Date de publication : 1 janvier 2015

Pagination : 158-161

ISBN : 978-2-919040-30-8

ISSN : $1142-852 X$

Référence électronique

Angéline Escafré-Dublet, «Aux origines de la revue Hommes \& Migrations », Hommes \& migrations [En ligne], 1309 | 2015, mis en ligne le 17 juillet 2015, consulté le 24 septembre 2020. URL : http:// journals.openedition.org/hommesmigrations/3101; DOI : https://doi.org/10.4000/ hommesmigrations.3101 


\section{COLLECTION}

\section{AUX ORIGINES DE LA REVUE HOMMES \& MIGRATIONS}

L'ASSOCIATION AMANA

ET LA CREATION DES CAHIERS NORD-AFRICAINS

(1945-1965)

ANGÉLINE ESCAFRÉ-DUBLET, maître de conférences en science politique, université Lumière Lyon-II, consultante scientifique pour "Repères", l'exposition permanente du Musée de l'histoire de l'immigration.

es origines de la revue Hommes \& Migrations Le trouvent dans le projet d'une association d'aide aux immigrés ayant pour but la promotion de la connaissance des populations du Maghreb en France, l'Amana'. Créée à Paris en 1945, l'association propose des cours d'alphabétisation aux travailleurs nord-africains et se dote également d'un service de publication, les Études sociales nord-africaines (ESNA). La production et la diffusion d'informations sont envisagées comme le complément nécessaire à l'activité d'alphabétisation : s'il faut familiariser ceux que l'on appelle alors les "Nord-Africains" à la vie en métropole, il faut aussi sensibiliser les acteurs sociaux aux problèmes qu'ils rencontrent et qui sont alors envisagés comme des problèmes "spécifiques". Deux publications parallèles et complémentaires sont créées et éditées par les ESNA : les Documents nord-africains (hebdomadaire qui reprend les articles parus dans la presse nationale et internationale) et les Cahiers nord-africains, où sont publiés des études et des dossiers entièrement consacrés à l'immigration nord-africaine en France. On compte 103 numéros des Cahiers nord-africains entre leur création en 1950 et l'année 1965 , date à laquelle ils deviennent la revue Hommes \& Migrations ${ }^{2}$. On trouve donc, aux origines de la revue, la volonté d'informer sur une expérience que l'on considère comme spécifique et méconnue, celle des immigrés en France. La production de connaissances et l'appréhension de ces questions ont évolué en fonction du contexte historique et politique qui entoure les phénomènes migratoires. 
des Pères Blancs, fondé en 1868 à Alger, et dont les activités s'étendaient à la Tunisie, à l'Afrique de l'Ouest et au Soudan. En plus de leurs activités d'évangélisation et d'éducation (au XIXe siècle, l'ordre comptait de nombreuses écoles privées en Algérie) $)^{3}$, les Pères Blancs ont produit un important travail ethnographique sur les populations colonisées 4 . En s'investissant dans l'association Amana à son arrivé à Paris, le père Ghys perpétue une tradition coloniale de travail ethnographique, tout autant qu'il la transforme et l'adapte au milieu métropolitain. En effet, il met à profit son expérience dans les colonies et les contacts qu'il a noués làbas, afin de poursuivre son investigation sur les populations nord-africaines en métropole. Il se constitue progressivement un statut d'expert sur l'immigration nord-africaine en France : l'expérience dans les colonies et l'investissement dans l'Amana lui donnent

Les Cahiers nord-africains, $\mathrm{n}^{\circ} 59$ (c) Hommes \& Migrations

\section{Une expertise sur l'immigration nord-africaine}

Dans l'immédiat après-guerre, l'association Amana et, à travers elle, son directeur, le père Jacques Ghys, se posent en intermédiaires indispensables entre deux mondes qui se connaissent peu. Amana veut d'ailleurs dire "confiance donnée" en arabe, ainsi qu'il est précisé dans les statuts de l'association. Ancien prêtre missionnaire en Tunisie, le père Ghys appartenait à l'ordre une garantie de proximité avec les immi-

grés nord-africains, la publication des Cahiers nord-africains et ses contacts dans l'administration lui permettent d'obtenir la reconnaissance institutionnelle nécessaire ${ }^{5}$.

L'expérience de l'Amana auprès de l'immigration nord-africaine intéressa en particulier une institution nationale créée pour venir en aide aux travailleurs coloniaux présents en métropole, le Fonds d'action sociale (Fas) ${ }^{6}$. À la création de ce fonds en 1959, son directeur, Michel Massenet, écrivait qu'en termes de soutien social et éducatif, l'Amana avait toujours été à "l'avant-garde de l'action à entreprendre" et bénéficiait d'une "expérience pratique considérable?". II suggéra que les autorités publiques

3. Bertrand Taithe, "Algerian orphans and colonial Chritianity in Algeria, 1866-1939", in French History, n²0, 2006, pp. $240-259$. 4. En 1985, Pierre Bourdieu expliquait comment le manque de méthode ethnographique dans la compilation d'informations des Pères Blancs sur les Algériens les avait amenés à laisser derrière eux un "trésor" que tous les ethnologues, y compris luimême, avaient pu utiliser. Voir Pierre Bourdieu, Mouloud Mammeri, “Du bon usage de l'ethnologie”, in Actes de la recherche en sciences sociales, vol. 5, n 150, 2003, p. 10. 5. "L'affirmation de la qualité d'expert, propre à chaque contexte, fait jouer l'expérience acquise, la reconnaissance institutionnelle, le rapport au pouvoir politique, la maitrise des savoirs ou, encore, la revendication d'une proximité avec le 'monde indigène' observé", Isabelle Backouche, "Devenir expert", in Genèses, n 70, 2008, p. 2.

6. Sur la création du Fas, voir Vincent Viet, La France immigrée. Construction d'une politique de l'immigration, 1938-1991, Paris, Fayard, 1991 ; Amelia Lyons, "Social welfare, French Muslims and decolonization in France: the case of the Fonds d'action sociale", in Patterns of Prejudice, vol. 43, n 1, 2009, pp. 65-89. 7. Michel Massenet, "Programme complémentaire pour 1959 à soumettre au Fas pour les travailleurs musulmans d’Algérie en métropole et pour leurs familles", 1er décembre 1958, 1977 O391, article 2 . 


\section{COLLECTION}

suivent son exemple. Plus tard, en 1960, le directeur demanda au père Ghys de lui suggérer une coordination entre les travailleurs nord-africains et les employeurs 8 .

D'autres organisations mirent en place des actions de type similaire, pour autant elles ne bénéficièrent pas d'un tel crédit auprès des autorités publiques. L'expérience pratique de l'Amana, appuyée par les publications des ESNA, ont donné au père Ghys le statut de conseiller dans la formulation des programmes en direction des migrants. En outre, dans le contexte de la guerre d'Algérie et de la surveillance des activités des Algériens, une importance primordiale fut accordée à l'approche psychologique9.

Les études proposées par les Cahiers nord-africains furent considérées comme pouvant servir à mieux connaître et à mieux comprendre les Algériens. Ils étaient lus par les préfets de police et leurs conseillers ; ils étaient également distribués auprès des officiers de police et cités dans les mémos administratifs ${ }^{10}$.

Enfin, les données recueillies par les Études sociales nord-africaines pour la publication des Cahiers nord-africains intéressèrent le monde de la recherche et, en particulier, le tout nouvel Institut national d'études démographiques (Ined), créé en 1945. Afin d'étudier les moyens qui pourraient contribuer à la croissance démographique de la France, les chercheurs de l'Ined développèrent des recommandations pour la sélection et l'adaptation des immigrés à la société française ${ }^{11}$. L'immigration nord-africaine était alors considérée comme moins connue.

La part des immigrés venus du Maghreb, et en particulier d'Algérie, augmenta à partir de la Seconde Guerre mondiale.En 1954, le nombre d'Algériens s'élevait à 212000 . C'était deux fois moins que les Italiens (508 000), mais déjà presque autant que les Espagnols (289 000) ou que les Polonais (269 000) ${ }^{12}$, des vagues migratoires plus anciennes qui avaient davantage été étudiées.

En 1946, quand Alfred Sauvy, le directeur de l'Ined, lança une recherche sur l'adaptation des Algériens à la société française, il proposa de consulter les "spécialistes des populations arabes".

C'est ainsi que les chercheurs de l'Ined contactèrent le père Ghys ${ }^{13}$. Les données furent collectées avec l'aide des ESNA. Les chercheurs de l'Ined profitèrent ainsi des connaissances de l'association et des contacts de son directeur. Cette collaboration avec le monde scientifique contribua à confirmer le directeur de l'Amana dans son statut d'expert sur l'immigration nord-africaine. En 1955, le père Ghys participait à une table ronde du Centre catholique pour les intellectuels français sur "les Nord-Africains en France", aux côtés du directeur de l'Ined, Alfred Sauvy, et du démographe Alain Girard'4.

\section{Le contexte \\ de la décolonisation}

Dans le contexte hautement politique de la décolonisation, le père Ghys et les associations caritatives d'aide aux migrants coloniaux en général adoptèrent un point de vue réformiste, pensant que la situation dans les colonies ne pourrait pas rester inchangée, même s'ils ne soutenaient pas l'action violente'5.

Ainsi, le travail de l'Amana et ses publications voulaient favoriser une meilleure compréhension des populations nord-africaines et améliorer leur situation. Cependant, il n'y avait jamais de critique directe du contrôle strict exercé par la police française sur les Algériens. En outre, le père Ghys appartenait à un réseau d'intellectuels qui 
réfléchissaient aux questions sociales avec une sensibilité catholique. La dimension religieuse de son engagement s'exprime dans sa conception de l'assimilation : dans cette perspective, l'islam constituait une barrière à une pleine assimilation. Cela, en vertu de la manière dont l'islam a été construit dans les colonies : comme un élément marquant une différence inhérente avec les coutumes européennes ${ }^{16}$. En métropole, cela voulait dire qu'une adaptation complète à la société française impliquait l'adoption de la langue et des coutumes françaises.

Tout le projet des Cahiers nord-africains visait à rendre plus compréhensible la vie des Nord-Africains à l'audience française. De ce point de vue, il était motivé par un fort sentiment de solidarité avec ces populations ${ }^{17}$.

Certains numéros étaient directement conçus pour fournir des repères sur les cultures islamiques et pour réduire les préjugés à l'encontre des musulmans.

Cependant, compte tenu des conditions de production et de diffusion des informations collectées, les Cahiers nord-africains permirent aussi de transmettre en métropole des pratiques spécifiques au contexte des colonies. L'association n'a pas pu réellement échapper à la situation politique de la France des années 1950. Pour le gouvernement français, l'aide sociale aux migrants était considérée comme une manière de maintenir le contrôle sur les populations coloniales et de les mettre à distance de l'activisme politique du Front de libération nationale (FLN) $)^{18}$. C'est pour cela qu'il plaça sa confiance dans des personnalités qui avaient été en contact avec ces populations dans les colonies, comme le père Ghys.

\section{Conclusion}

Loin de représenter une rupture avec la conception coloniale de l'assimilation, les activités de l'Amana signifièrent plutôt une transition de la mission civilisatrice de l'empire français vers l'impératif culturel d'intégration dans la Ve République. Les activités de l'Amana continuèrent dans les années 1960. En 1965, les Cahiers Nord-Africains furent rebaptisés Hommes \& Migrations. L'accession à l'indépendance de l'Algérie ne justifiait plus l'existence d'un Fonds d'aide sociale aux travailleurs musulmans présents en métropole, puisqueceux-ci n'étaient plus des sujets coloniaux relevant de l'administration française. Pourtant, l'idée d'une administration structurant l'aide sociale fournie aux travailleurs immigrés présents en France perdura ${ }^{19}$. À la demande de cette administration, les associations caritatives d'aide aux migrants coloniaux furent enjointes d'étendre leurs activités à l'ensemble des étrangers. Selon cette logique, les Cahiers nord-africains cessèrent de s'intéresser prioritairement aux populations venues d'Afrique du Nord et devinrent Hommes \& Migrations, pour couvrir des sujets qui touchaient tous les immigrés, quelles que soient leurs origines. L'idée, cependant, que les immigrés devaient faire l'objet d'une attention spécifique, car leurs besoins étaient mal connus, demeura. Les origines de la revue Hommes \& Migrations témoignent donc de cette période de transition qui fit passer la France d'une nation coloniale à un pays d'immigration et qui fut cruciale dans la définition de son appréhension des immigrés.

\footnotetext{
11. Sur les études réalisées par l'Ined sur les immigrés au début des années 1950, voir Angéline Escafré-Dublet, Lionel Kesztenbaum, “Mesurer l'intégration des immigrés. Genèses et histoire des enquêtes Girard-Stoetzel, 1945-1953", in Genèses, $n^{\circ} 84,2011.12$. Recensement, 1954, Insee. 13. Alfred Sauvy, compte rendu de réunion, 4 avril 1946, archives de I'Ined, A 3118. 14. Emmanuel Blanchard, op. cit., p. 227. 15. Florence Denis, “Entre mission et développement : I'association Ad Lucem et le laïcat missionnaire (1945-1957)", in Mouvement social, n 177, 1996, pp. 29-48. 16. Paul Silverstein, Algeria in France, Transpolitics, Race, and Nation, Bloomington, Indiana University Press, 2004. 17. Sur la question de la solidarité au fondement du projet de l'Amana, voir Angéline Escafré-Dublet, "Aid, activism and the State in post-war France : Amana, a charity organisation for colonial migrants, 1945-1962", in Journal of Modern European History, n¹2, 2014, pp. 247-262. 18. Amelia Lyons, The Civilizing Mission in the Metropole: Algerian Families and the French Welfare, Stanford University Press, 2013 19. Vincent Viet, op.cit.
} 